\title{
Mortality Risk Stratification After Traumatic Brain Injury and Hazard of Death With Titrated Hypothermia in the Eurotherm3235Trial
}

\author{
Peter J. D. Andrews, $\mathrm{MD}^{1}$; Aryelly Rodriguez, $\mathrm{PhD}^{2}$; Peter Suter, $\mathrm{MD}^{3}$; Claire G. Battison, BSc \\ Jonathan K. J. Rhodes, $\mathrm{MD}^{4}$; Irene Puddu, $\mathrm{MD}^{5}$; Bridget A. Harris, $\mathrm{PhD}^{4}$
}

\begin{abstract}
${ }^{1}$ Centre for Clinical Brain Sciences, University of Edinburgh, Edinburgh, United Kingdom.

${ }^{2}$ Centre for Population Health Sciences, The University of Edinburgh, Medical School, Edinburgh, United Kingdom.

${ }^{3}$ Department of Anaesthesiology, Pharmacology and Surgery Intensive Care, University of Geneva, Genève, Switzerland.

${ }^{4}$ Department of Anaesthesia, Critical Care and Pain Medicine, University of Edinburgh, Edingburgh, United Kingdom.

${ }^{5}$ Department of Anaesthesia and Critical Care, University of Turin, Turin, Italy. The views and opinions expressed therein are those of the authors and do not necessarily reflect those of the National Institute for Health Research Health Technology Assessment program or the Department of Health. Current Controlled Trials number, ISRCTN34555414.
\end{abstract}

Supplemental digital content is available for this article. Direct URL citations appear in the printed text and are provided in the HTML and PDF versions of this article on the journal's website (http://journals.lww.com/ ccmjournal).

This project was funded by the National Institute for Health Research Health Technology Assessment program (project number 11/01/30). The European Society of Intensive Care Medicine funded the pilot phase (11). Professor Andrews (Chief Investigator) disclosed that the European Society of Intensive Care Medicine (ESICM) funded the pilot phase; he received funding from lecturing fees for BARD and Integra Neuroscience, and he received support for article research from the National Institute for Health Research (NIHR) Health Technology Assessment (HTA) program. Sponsored by The University of Edinburgh and National Health Service Lothian. Dr. Rodriguez's institution received funding from NIHR HTA research grant, and she received support for article research from the NIHR. Ms. Battison received support for article research from the NIHR HTA and ESICM. Dr. Rhodes disclosed fellowship support from National Health Service Research Scotland; he received funding from BARD (speakers fees for educational meetings) and Integra (speakers bureau). Dr. Puddu received support for article research from the NIHR HTA. Dr. Harris received support for article research from the NIHR HTA (U.K. Government funding) and from ESICM; her institution received funding from Edinburgh and Lothians Health Foundation, NHS Research Scotland, and from NIHR HTA. Dr. Suter has disclosed that he does not have any potential conflicts of interest.

For information regarding this article, E-mail: p.andrews@ed.ac.uk

Copyright (c) 2017 The Author(s). Published by Wolters Kluwer Health, Inc. on behalf of the Society of Critical Care Medicine and Wolters Kluwer Health, Inc. This is an open-access article distributed under the terms of the Creative Commons Attribution-Non Commercial-No Derivatives License 4.0 (CCBY-NC-ND), where it is permissible to download and share the work provided it is properly cited. The work cannot be changed in any way or used commercially without permission from the journal.

DOI: 10.1097/CCM.0000000000002376
Objectives: Hypothermia reduces intracranial hypertension in patients with traumatic brain injury but was associated with harm in the Eurotherm3235Trial. We stratified trial patients by International Mission for Prognosis and Analysis of Clinical Trials in [Traumatic Brain Injury] (IMPACT) extended model sum scores to determine where the balance of risks lay with the intervention.

Design: The Eurotherm3235Trial was a randomized controlled trial, with standardized and blinded outcome assessment. Patients in the trial were split into risk tertiles by IMPACT extended model sum scores. A proportional hazard analysis for death between randomization and 6 months was performed by intervention and IMPACT extended model sum scores tertiles in both the intention-to-treat and the per-protocol populations of the Eurotherm3235Trial.

Setting: Forty-seven neurologic critical care units in 18 countries. Patients: Adult traumatic brain injury patients admitted to intensive care who had suffered a primary, closed traumatic brain injury; increased intracranial pressure; an initial head injury less than 10 days earlier; a core temperature at least $36^{\circ} \mathrm{C}$; and an abnormal brain CT.

Intervention: Titrated Hypothermia in the range $32-35^{\circ} \mathrm{C}$ as the primary intervention to reduce raised intracranial pressure.

Measurements and Main Results: Three hundred eighty-six patients were available for analysis in the intention-to-treat and 257 in the per-protocol population. The proportional hazard analysis (intention-to-treat and per-protocol populations) showed that the treatment effect behaves similarly across all risk stratums. However, there is a trend that indicates that patients in the low-risk group could be at greater risk of suffering harm due to hypothermia.

Conclusions: Hypothermia as a first line measure to reduce intracranial pressure to less than $20 \mathrm{~mm} \mathrm{Hg}$ is harmful in patients with a lower severity of injury and no clear benefit exists in patients with more severe injuries. (Crit Care Med 2017; 45:883-890)

Key Words: IMPACT extended prediction model; randomized controlled trial; therapeutic hypothermia; traumatic brain injury 
$\mathrm{E}$ xperimental studies of hypothermia delivered after all forms of acute brain injury show neurologic benefit (infarct volume and neurologic behavior scores), even when the induction of hypothermia is delayed after injury and temperature reduction is modest (1). Therapeutic hypothermia $(\mathrm{TH})$ has previously been tested after traumatic brain injury (TBI) to assess prophylactic neurologic protection (2).

The Eurotherm3235Trial was a large randomized controlled trial that tested $\mathrm{TH}$ as the primary intervention (after stage I interventions) to reduce intracranial pressure (ICP) below $20 \mathrm{~mm} \mathrm{Hg}$ after TBI compared with standard care (control). In this way, TH was delivered in a dosage (depth and duration) dictated by a biomarker of the injury process, ICP, to reduce the burden of possible complications in milder injury and keeping colder temperatures for more severe injury/brain swelling. The intention-to-treat (ITT) analysis showed higher mortality and worse outcomes with TH compared with standard care, and the trial was stopped early because of likely futility and the potential for harm (3).

Postpublication sentiment has considered that TH could still be used in the TBI patients with refractory elevated ICP (4).

TBI is a heterogeneous disease with respect to cause, pathology, severity, and prognosis. This causes considerable uncertainty in the expected outcome of individual patients. Prognostic models can be used to combine different characteristics of an individual patient to predict outcome. The choice of model depends upon the clinical setting and case-mix of the population under study. The International Mission for Prognosis and Analysis of Clinical Trials in TBI (IMPACT) models were developed on patients with moderate and severe TBI and mostly from high-income countries $(5,6)$. Given the data collected in Eurotherm3235Trial, the IMPACT extended model sum score (IEMSS) was selected as the most representative calculation to determine the baseline risk score for the patients, in order to assess the relationship between severity and the intervention effect.

The aim of this analysis is to assess the relationship between IEMSS and the intervention effect.

\section{MATERIALS AND METHODS}

On May 29, 2013, the Data Monitoring Committee (DMC) requested an additional analysis of the primary endpoint and mortality by IEMSS tertiles as a further participant safeguard. The first such IEMSS analysis was delivered on July 24, 2013, and after review, the DMC members decided to keep the subgroup analysis by IEMSS permanently in the DMC report. This analysis was not included in the final trial statistical analysis plan (SAP) and has not been published before but may provide important information to clinicians about the choice of therapy for ICP reduction.

\section{Interventions and Study Procedures}

The Eurotherm3235Trial was a pragmatic, randomized controlled trial, with standardized and blinded outcome assessment (7). Intracranial hypertension was defined in accordance with the Brain Trauma Foundation (BTF) guidelines, 2007 (8).

In brief, eligible participants with ICP greater than $20 \mathrm{~mm} \mathrm{Hg}$ were randomized to either standard care (including mechanical ventilation and increased sedation) with osmotherapy (control group) or standard care with titrated $\mathrm{TH}$ as the primary intervention to reduce ICP less than $20 \mathrm{~mm}$ Hg. Hypothermia was maintained for at least 48 hours and continued for as long as was necessary to maintain ICP less than or equal to $20 \mathrm{~mm} \mathrm{Hg}$, core temperature was to remain within the limits $32-35^{\circ} \mathrm{C}$. Rewarming was then considered provided the ICP remained less than or equal to $20 \mathrm{~mm} \mathrm{Hg}$.

Ethical approval was obtained from Scotland A Research Ethics Committee (REC) as the lead REC (09/MRE00/34) and additionally Bradford REC (09/H1302/44) and ethics committees in a further 14 countries. Due to their incapacitated state, it was not possible to obtain consent directly from potential participants. Informed consent was therefore sought from each eligible patient's nearest relative or another person designated to give consent on the patient's behalf.

\section{Outcomes}

The primary outcome measure was the Extended Glasgow Outcome Scale (GOSE) score at 6 months after injury. The GOSE was assessed by a structured questionnaire (by post or interview), and a blinded investigator scored all outcomes according to a standardized approach (9). The 6-month mortality was a secondary outcome.

\section{Per-Protocol (PP) Population}

The PP population comprises those members of the ITT population who completed the study without a major protocol violation and who complied adequately with the administered intervention. Intervention compliance was decided using the following strategy and is described in the trial's SAP:

Step 1. If a patient was allocated to an intervention, then they should have received the allocated intervention.

Step 2. If a patient was allocated to control group, then their core temperature should be strictly equal to or greater than $36^{\circ} \mathrm{C}$ for at least $80 \%$ of the 48 possible temperature observations for the first 48 hours from randomization or until death (for whichever event occurred first).

Step 3. If a patient was allocated to hypothermia group, then their core temperature should be strictly greater than or equal to $32^{\circ} \mathrm{C}$ and strictly less than $35^{\circ} \mathrm{C}$ for at least $80 \%$ of the 44 possible temperature observations from 4 hours after hypothermia started until the first 48 hours from randomization or until death (for whichever event occurred first). No barbiturate infusion was permitted within the first 48 hours from randomization.

\section{IEMSS}

Sum scores were calculated for all participants using the IMPACT extended model (10) using age, motor score, pupillary reactivity, Marshall CT classification, presence of traumatic subarachnoid hemorrhage on CT, and extradural hematoma on CT (Fig. S1, Supplemental Digital Content 1, http://links. lww.com/CCM/C471-legend, Supplemental Digital Content 2, http://links.lww.com/CCM/C472). These data were routinely collected in all subjects and available in the Case Report Form. Hypoxia and hypotension values were not recorded in the Eurotherm3235Trial and were assigned "0" points (10). 
The IEMSS was ordered and split into tertiles in order to generate a baseline risk profile for each patient (low, medium, and high). The population for the calculation of the risk and subsequent tertile allocation is the ITT population, so when data are filtered by compliance (i.e., PP population), the tertiles will not necessarily remain equal in terms of numbers in each. The boundaries of the (statistical) tertiles were adjusted to group patients with the same risk score (IEMSS) into the same risk category, that is, if a patient was in the low-risk category but had an IEMSS and the same risk calculation as any patient in the medium category, then they were reallocated to medium-risk tertile. Similar adjustments were made for medium-risk participants, in a way that always made the risk tertile worse instead of better.

The probabilities of 6-month outcomes were calculated as (10):

Probability of mortality $=$

$1 /(1+\exp (-2.98+0.256 *($ sum score extended model $)))$

Probability of unfavorable $=$

$1 /\left(1+\exp \left(-2.10+0.276^{\star}(\right.\right.$ sum score extended model $\left.\left.)\right)\right)$

\section{Statistical Methods}

The distribution of the 6-month GOSE between the groups (hypothermia vs control) by IEMSS was compared using ordinal regression, adjusting for time from injury ( $<12 \mathrm{vs} \geq 12 \mathrm{hr}$ ), including an interaction term between IEMSS and the intervention. Also a nonadjusted model was fitted. The eight-point GOSE was collapsed to six categories by pooling death with vegetative state and lower severe disability. Also, the conventional dichotomized split of the GOSE as lower moderate disability or better (favorable) versus upper severe disability or worse (unfavorable) was analyzed. For the difference in mortality, Cox proportional hazards regression (fitting intervention, IEMSS, and an interaction term) was used, and its assumptions were verified by visual inspection of the survival curves (11). Analyses were performed with SAS software, version 9.4 (SAS Institute, Cary, NC). A level of statistical significance $p$ value of less than 0.05 was used.

\section{RESULTS}

Data from 386 patients were available for analysis for the ITT population. Data from 257 patients were available for analysis for the PP population (Table 1). Demographic and baseline characteristics by the IEMSS tertiles are presented in Tables $\mathbf{S 1}$ and S2 (Supplemental Digital Content 2, http://links.lww.com/ $\mathrm{CCM} / \mathrm{C} 472)$ for the ITT population. The GOSE and mortality rate distribution by intervention are presented in Figure 1. The analysis for the GOSE and mortality rate showed no significant interaction effect between the intervention and the IEMSS tertiles for the ITT and PP populations (Figs. 2 and $\mathbf{3}$ ).

The adjusted and nonadjusted analysis for the collapsed and dichotomized GOSE showed no statistically significant difference between the intervention for each IEMSS tertile. The odds ratio favored "control" over hypothermia in all comparisons (Fig. 2).

The proportional hazard analysis for death between randomization and 6 months by IEMSS tertile and intervention behaved similarly across high- and medium-risk stratums, but there is a change in the magnitude of the effect in the lower risk cohort (Fig. 3).

The Glasgow Coma Scale (GCS) sum score at admission was less than or equal to 8 in approximately $50 \%$ of the patients in lower and medium-risk tertiles and just over $20 \%$ of patients were characterized as having a mild TBI (GCS, 13-15) on hospital admission. These were patients who suffered secondary deterioration, and a greater proportion of patients had neurosurgery (CT Marshall score V) in the medium- and high-risk tertiles but the proportions were similar, 25\% and 19\%, respectively (Table S2, Supplemental Digital Content 2, http://links. lww.com/CCM/C472).

Intracranial pathology (Table S3, Supplemental Digital Content 2, http://links.lww.com/CCM/C472): More extradural hematomas were evident in the low-risk tertiles compared with medium- and high-risk tertiles (as expected), with more diffuse axonal injury in the high-risk ITT cohorts and more traumatic subarachnoid hemorrhage in high (and medium) PP cohorts.

Intracranial neurosurgery (Table S4, Supplemental Digital Content 2, http://links.lww.com/CCM/C472): More extradural

\section{TABLE 1. Eurotherm3235Trial Protocol Compliance}

\begin{tabular}{|c|c|c|c|c|}
\hline Eurotherm3235Trial Compliance & Categories & $\begin{array}{c}\text { Allocated } \\
\text { Hypothermia }\end{array}$ & $\begin{array}{l}\text { Allocated } \\
\text { Control }\end{array}$ & Overall \\
\hline Intention-to-treat population $(n, \%)$ & All subjects & $195(100)$ & $192(100)$ & $387(100)$ \\
\hline \multirow[t]{2}{*}{ Per-protocol population $(n, \%)$} & No & $70(35.9)$ & $60(31.3)$ & $130(33.6)$ \\
\hline & Yes & $125(64.1)$ & $132(68.7)$ & $257(66.4)$ \\
\hline \multirow[t]{3}{*}{ Intervention compliance $(n, \%)$} & Missing data & 2 & 0 & 2 \\
\hline & Yes & $125(64.8)$ & $132(68.8)$ & $257(66.8)$ \\
\hline & No & $68(35.2)$ & $60(31.3)$ & $128(33.2)$ \\
\hline
\end{tabular}

Treatment compliance to determine the per-protocol group was decided using a strategy that is described in the text and in more detail in the trial's statistical analysis plan. 

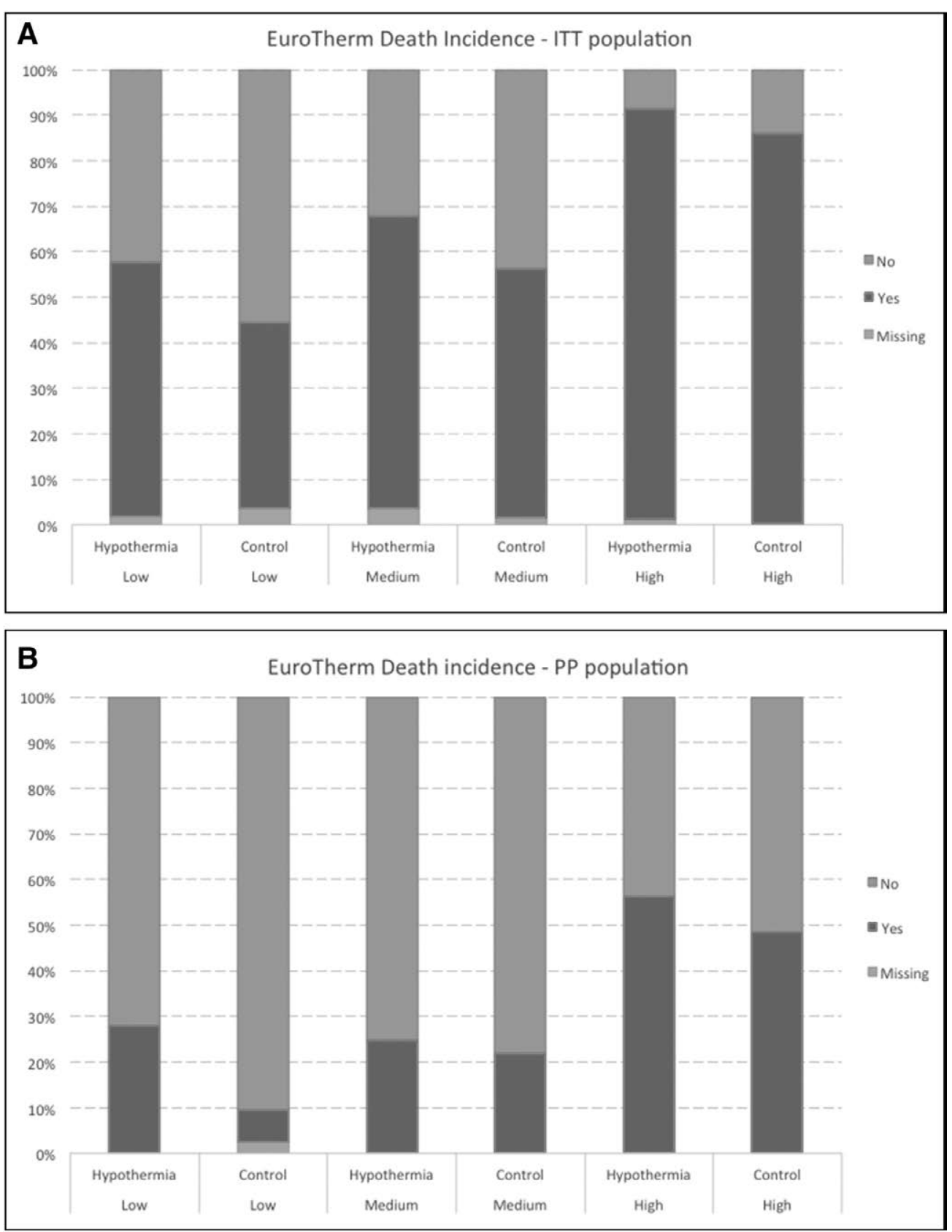

Figure 1. Bar charts showing the distribution of mortality and dichotomized Glasgow Outcome Scale Extended (GOSE) into favorable and unfavorable outcomes, by intention-to-treat (ITT) and per-protocol (PP) participant populations (four bar charts to one figure). A, Occurrence rate of death, ITT population. B, Occurrence rate of death, PP population. C, Glasgow Outcome Scale Extended, ITT population. D, Glasgow Outcome Scale Extended, PP population.

hematoma evacuations occurred in the low-risk tertiles, but the trend was otherwise for more operative interventions in the medium- and high-risk tertiles.

\section{DISCUSSION}

In summary, the analysis split by IEMSS tertiles showed a general trend toward harm for both the ITT and the PP populations with hypothermia. Furthermore, when the IEMSS was used for risk stratification of the PP population, the largest intervention effect was evident in the low-risk IEMSS group of patients who were theoretically least likely to suffer death $(9 \%$ mean probability) and unfavorable outcomes (18\% mean probability) (Table 2). Consequently, it was the lowest risk of death group where the (potentially adverse) intervention effect was most evident and this intervention effect was greatest when the intervention was delivered according to the protocol (Fig. 3) (Fig. S2, Supplemental Digital Content 3, http://links. lww.com/CCM/C473-legend, Supplemental Digital Content 2, http://links.lww.com/ $\mathrm{CCM} / \mathrm{C} 472)$. The patients with the lowest IMPACT scores are the youngest patients in the trial and when ICP is difficult to control at stage II, and because of the perception they have most to lose, they are the group in whom hypothermia is often considered as a stage III (last ditch) intervention.

Analysis of intracranial lesion(s) and neurosurgery intervention(s) did not reveal imbalances that could account for these findings. Therefore, the results of this trial could be due to either a biological effect of the hypothermia intervention or an imbalance in cointerventions, including a restriction in the use of barbiturate infusion with hypothermia.

Prediction models are important tools for heterogeneity adjustment in clinical trials and for the evaluation of the quality of care delivered to patients with TBI. The Corticosteroid Randomisation After Significant Head Injury (CRASH) and IMPACT prognostic models have been developed using contemporary approaches to large datasets $(5,10$, $12)$ and were externally validated reciprocally $(10,13,14)$. The IMPACT model was validated against the CRASH trial data that similarly had no data on hypoxia and hypotension. We therefore followed an established methodology that demonstrated good agreement between the IMPACT EMSS and CRASH data for assessment of severity of injury.

Although randomization was extended to 10 days following review of the pilot data, the admission characteristics were used for calculation of baseline prognostic risk. The demographics 


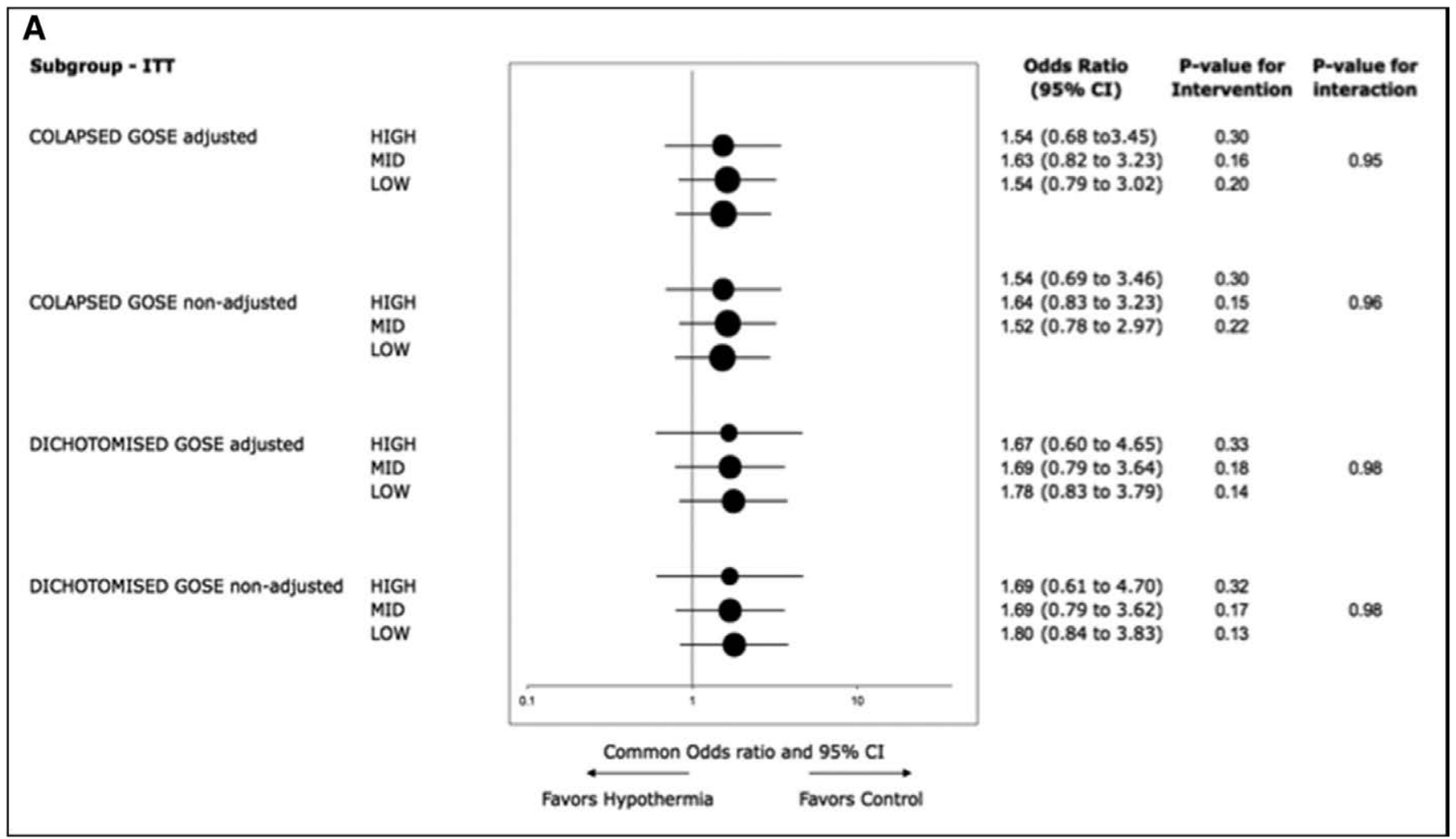

B

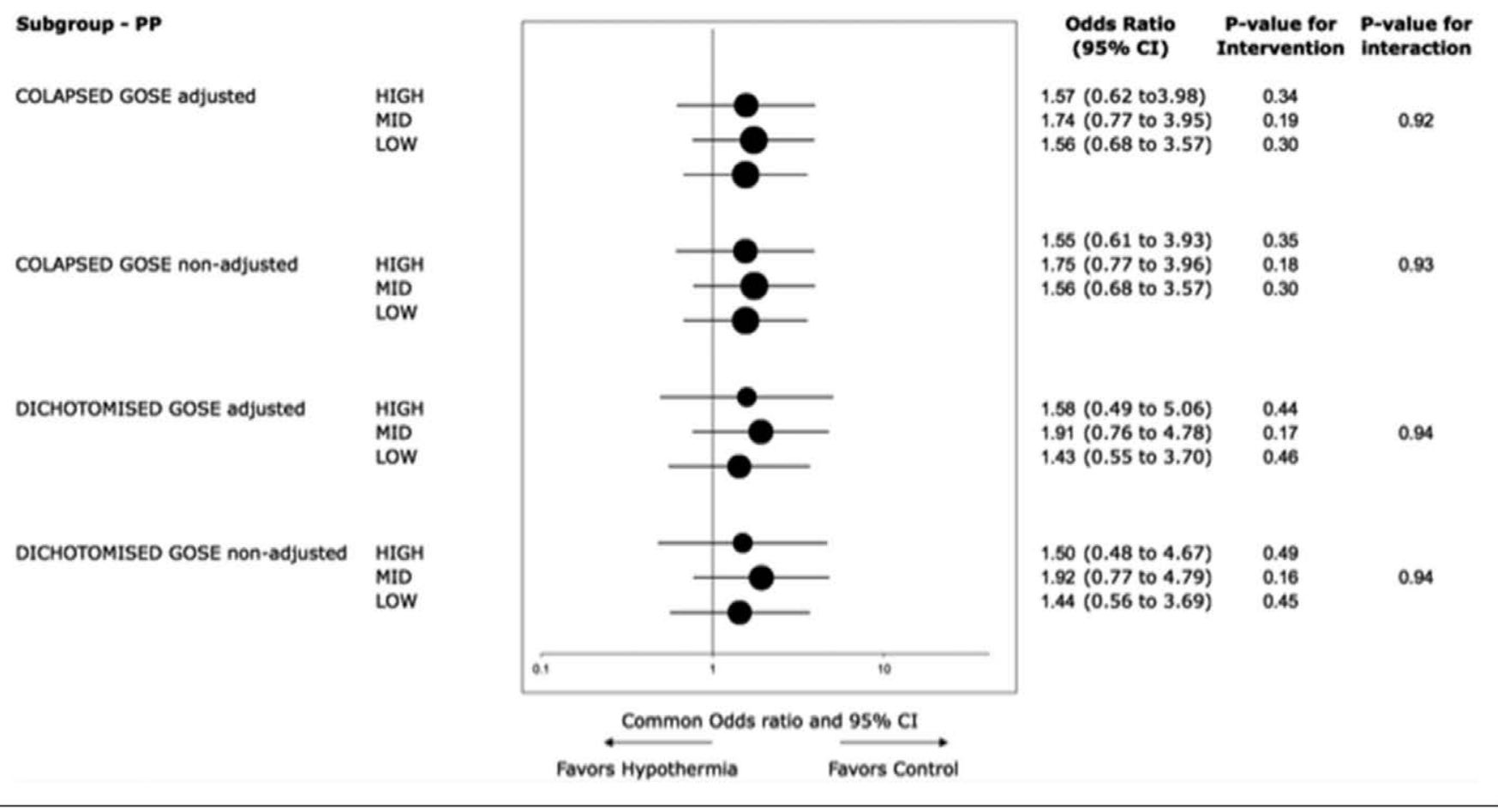

Figure 2. Forest plot of the Eurotherm3235Trial Glasgow Outcome Scale Extended (GOSE) by intervention and International Mission for Prognosis and Analysis of Clinical Trials in [Traumatic Brain Injury] (IMPACT) Extended Model Sum Score (IEMSS). Data are analyzed by 1) collapsed ordinal analysis of the GOSE with death, vegetative state, and lower severe disability combined so as not to give credit for severely impaired survival and 2) a dichotomized analysis. Both analyses are presented with adjusted and nonadjusted (for baseline risk) outcomes. A, Intention-to-treat (ITT) Population. GOSE statistical analysis by intervention and IEMSS. B, Per-protocol (PP) population. GOSE statistical analysis by intervention and IEMSS. 


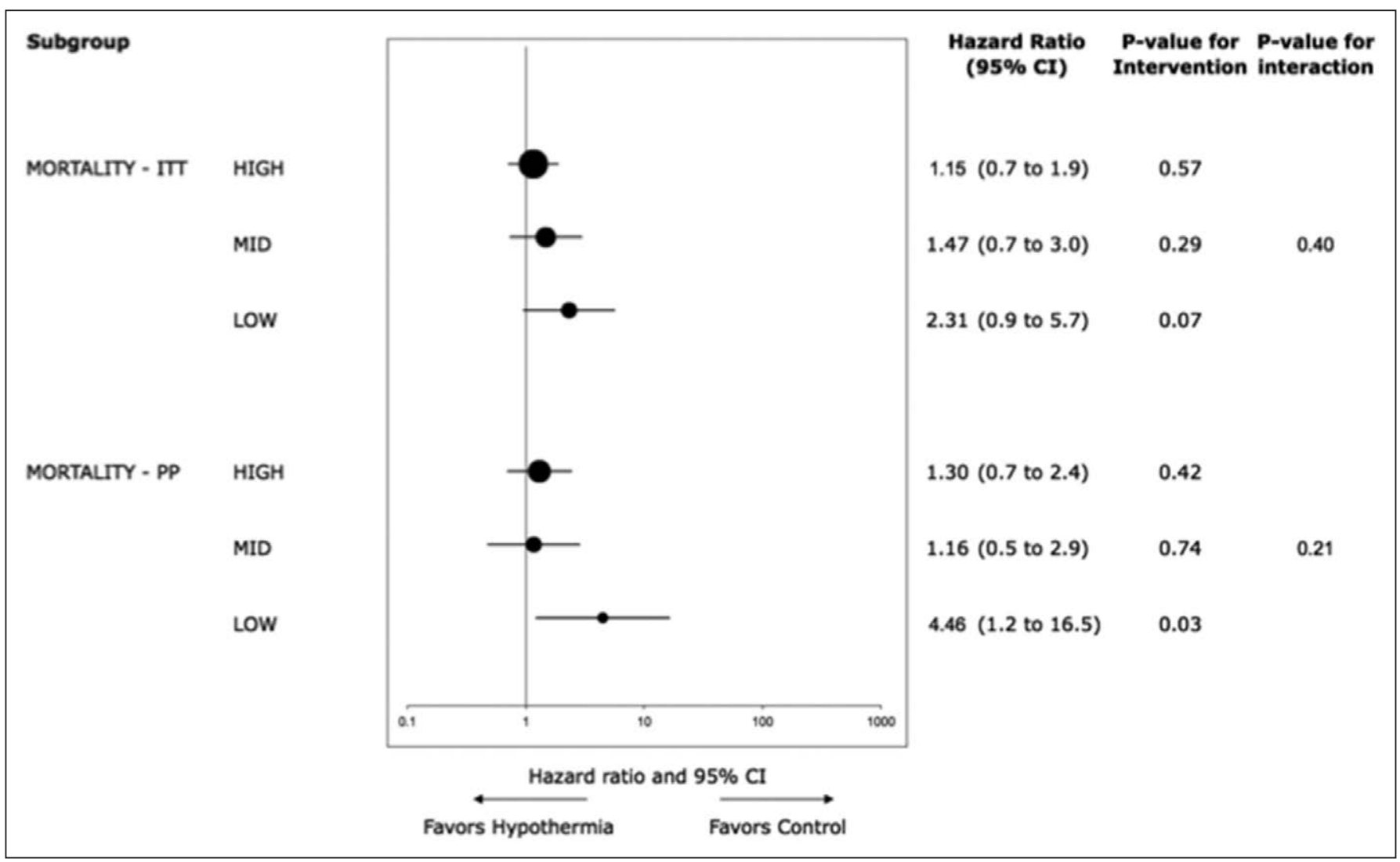

Figure 3. Forest plot of the Eurotherm3235Trial mortality by intervention and International Mission for Prognosis and Analysis of Clinical Trials in [Traumatic Brain Injury] (IMPACT) Extended Model Sum Score. Data are presented as a proportional hazard analysis for death. ITT = intention-to-treat, PP = per-protocol.

of these patients were similar to IMPACT, and the model is therefore applicable to the Eurotherm3235Trial cohort.

There were no important differences between the three tertiles for ICP control at 20 or $25 \mathrm{~mm} \mathrm{Hg}$ thresholds or for new infection. When the PP patients were examined, the effect of hypothermia was to significantly increase mortality in the lowest risk tertile. The better outcome potential of the lowest risk tertile unmasked the harm of the intervention. However, no benefit was evident in the medium- and high-risk tertiles in any analyses (adjusted and nonadjusted) and the odds ratio always favored control.

The Eurotherm3235Trial protocol resulted in the use of cooling at a relatively early stage for ICP control, once the first line measures of sedation, positioning, and optimization of arterial blood gas tensions had failed. One criticism of the trial is that in many ICUs hypothermia is applied later, once other measures such as paralysis and hypertonic treatments have failed. The rational for this trial design was to reduce the confounding effects of multiple, poorly evaluated hypertonic therapies (where the benefits and harms are not known) in the hypothermia group. The restriction of cooling to cases in which ICP control is more refractory to treatment might in effect be reserving it for those with a predicted IEMSS high risk of death. Based on these data our analysis did not demonstrate a benefit to this tertile.

At the inception of the Eurotherm3235Trial, we considered that if TH was targeted as a biomarker of the injury process, this would lead to a better balance of depth and duration of hypothermia to injury severity. Raised ICP was considered an appropriate biomarker of TBI as it is a life-threatening complication of TBI and can result in compromised cerebral circulation, brain stem compression, and brain death. Increased ICP is strongly associated with poorer outcomes after TBI and therefore has been regarded as a target for therapy (8).

ICP monitoring is generally viewed as the cornerstone of care in these patients and is recommended in all modern guidelines for treatment of TBI. However, studies showing beneficial effects of this approach are lacking. The newly released fourth edition of the BTF guidelines (15) recommend treating ICP greater than $22 \mathrm{~mm} \mathrm{Hg}$ because values above this level are associated with increased mortality. An important finding of the Eurotherm study was the efficacy of contemporary critical care management to control ICP after TBI. The Eurotherm3235Trial screening log showed ICP was controlled with first line measures in $84 \%$ of patients.

What is the benefit of ICP monitoring over and above clinical examination and cross-sectional imaging? Chesnut et al (16) and Cremer et al (17) showed no outcome benefit from ICP monitoring and an increased length of ICU and hospital stay. Previous studies that have evaluated interventions that reduce ICP are neutral or show a trend toward harm. These include Decompressive Craniectomy in Diffuse Traumatic Brain Injury (DECRA), CRASH, Decompressive Craniectomy for Traumatic Intracranial Hypertension (RescueICP), and previous studies of TH (18-22). 


\section{TABLE 2. Predicted Mortality and Predicted Dichotomized Glasgow Outcome Score Extended Probabilities by International Mission for Prognosis and Analysis of Clinical Trials in [Traumatic Brain Injury] Extended Model Sum Score Divided into Three Tertiles}

International Mission for Prognosis

and Analysis of Clinical Trials in

[Traumatic Brain Injury] Extended

Model Sum Scores Tertile

Allocated

Intervention
Lower $95 \%$ Cl

for Mean
Upper $95 \%$ Cl

for Mean
SD

ITT population probability of mortality ${ }^{a}$

$\begin{array}{lllllrr}\text { Low } & \text { Hypothermia } & 57 & 0.100 & 0.093 & 0.107 & 0.025 \\ \text { Mid } & \text { Control } & 56 & 0.092 & 0.086 & 0.099 & 0.023 \\ & \text { Hypothermia } & 56 & 0.187 & 0.179 & 0.196 \\ \text { High } & \text { Control } & 64 & 0.192 & 0.184 & 0.199 & 0.031 \\ & \text { Hypothermia } & 82 & 0.439 & 0.405 & 0.473 \\ & \text { Control } & 72 & 0.444 & 0.411 & 0.478\end{array}$

ITT population probability of unfavorable outcome

\begin{tabular}{|c|c|c|c|c|c|c|}
\hline \multirow[t]{2}{*}{ Low } & Hypothermia & 57 & 0.220 & 0.206 & 0.234 & 0.053 \\
\hline & Control & 56 & 0.204 & 0.192 & 0.217 & 0.048 \\
\hline \multirow[t]{2}{*}{ Mid } & Hypothermia & 56 & 0.383 & 0.369 & 0.397 & 0.052 \\
\hline & Control & 64 & 0.390 & 0.377 & 0.403 & 0.051 \\
\hline \multirow[t]{2}{*}{ High } & Hypothermia & 82 & 0.677 & 0.648 & 0.707 & 0.135 \\
\hline & Control & 72 & 0.686 & 0.657 & 0.715 & 0.124 \\
\hline \multicolumn{7}{|c|}{ P population probability of mortality } \\
\hline \multirow[t]{2}{*}{ Low } & Hypothermia & 32 & 0.092 & 0.083 & 0.102 & 0.026 \\
\hline & Control & 41 & 0.092 & 0.085 & 0.100 & 0.02 \\
\hline \multirow[t]{2}{*}{ Mid } & Hypothermia & 40 & 0.186 & 0.176 & 0.197 & 0.033 \\
\hline & Control & 41 & 0.190 & 0.180 & 0.199 & 0.030 \\
\hline \multirow[t]{2}{*}{ High } & Hypothermia & 53 & 0.426 & 0.385 & 0.467 & 0.148 \\
\hline & Control & 50 & 0.453 & 0.410 & 0.495 & \\
\hline
\end{tabular}

PP population probability of unfavorable outcome

\begin{tabular}{lllllrr} 
Low & Hypothermia & 32 & 0.184 & 0.184 & 0.224 & 0.056 \\
Mid & Control & 41 & 0.189 & 0.186 & 0.221 & 0.050 \\
& Hypothermia & 40 & 0.363 & 0.363 & 0.398 \\
High & Control & 41 & 0.372 & 0.372 & 0.403 & 0.049 \\
& Hypothermia & 53 & 0.631 & 0.631 & 0.131 \\
& Control & 50 & 0.656 & 0.656 & 0.126 \\
\hline
\end{tabular}

ITT = intention-to-treat, $\mathrm{PP}=$ per-protocol.

aProbability calculated using the International Mission for Prognosis and Analysis of Clinical Trials in [Traumatic Brain Injury] "extended model," Variables hypoxia and hypotension were set to "no" for all patients.

Historically, the more commonly investigated use of hypothermia following TBI has been for neuroprotection, the intervention being applied as soon as is practicable after injury. One such multicenter RCT is ongoing (Prophylactic Hypothermia Trial to Lessen Traumatic Brain Injury [POLAR-RCT]). The larger RCTs such as National Acute Brain Injury Study: Hypothermia (NABIS H) II (20), and B-HYPO (24) failed to demonstrate a neuroprotective effect of hypothermia and many reasons have been suggested for this.

The Eurotherm3235Trial was a study of the use of hypothermia to control ICP. We tested a widely used intervention to reduce ICP below an internationally recognized threshold and that strategy led to significantly poorer outcomes, increased mortality and, when assessed on a PP basis, harmed 
participants considered at lowest risk of death, when compared with medical care that aimed to do the same. Stratification of patients according to the IEMSS (10) showed that the burden of harm was in the low risk of poor outcome and/or death cohort, with no evidence of benefit across any severity tertile.

\section{Implications of All the Available Evidence}

The four largest trials of TH after TBI all show evidence of increased mortality $(3,20,21,23)$ with TH. Therefore, despite optimistic Systematic Reviews (2), TH should not be used after TBI, for neuroprotection or to reduce ICP (4) outside of clinical trials.

\section{CONCLUSIONS}

Hypothermia as a first line measure to reduce ICP to less than $20 \mathrm{~mm} \mathrm{Hg}$ is potentially harmful in patients with a lower severity of injury assessed by IEMSS and no clear benefit exists in patients with more severe injuries. We recommend against the use of hypothermia after TBI and in particular in the context of ICP reduction.

\section{REFERENCES}

1. van der Worp HB, Sena ES, Donnan GA, et al: Hypothermia in animal models of acute ischaemic stroke: A systematic review and metaanalysis. Brain 2007; 130:3063-3074

2. Crossley S, Reid J, McLatchie R, et al: A systematic review of therapeutic hypothermia for adult patients following traumatic brain injury. Crit Care 2014; 18:R75

3. Andrews PJ, Sinclair HL, Rodriguez A, et al; Eurotherm3235 Trial Collaborators: Hypothermia for intracranial hypertension after traumatic brain injury. N Engl J Med 2015; 373:2403-2412

4. Andrews PJ, Harris BA, Murray GD: Hypothermia for intracranial hypertension after traumatic brain injury. N Engl J Med 2016; 374:1385

5. Marmarou A, Lu J, Butcher I, et al: IMPACT database of traumatic brain injury: Design and description. J Neurotrauma 2007; 24: 239-250

6. Majdan M, Lingsma HF, Nieboer D, et al: Performance of IMPACT, $\mathrm{CRASH}$ and Nijmegen models in predicting six month outcome of patients with severe or moderate TBI: An external validation study. Scand J Trauma Resusc Emerg Med 2014; 22:68

7. Andrews PJ, Sinclair HL, Battison CG, et al; Eurotherm3235Trial collaborators: European society of intensive care medicine study of therapeutic hypothermia $\left(32-35^{\circ} \mathrm{C}\right)$ for intracranial pressure reduction after traumatic brain injury (the Eurotherm3235Trial). Trials 2011; 12:8

8. Brain Trauma Foundation; American Association of Neurological Surgeons; Congress of Neurological Surgeons; Joint Section on Neurotrauma and Critical Care, AANS/CNS, Bratton SL, Chestnut RM, Ghajar J, et al: Guidelines for the management of severe traumatic brain injury. VIII. Intracranial pressure thresholds. I Neurotrauma 2007; 24 (Suppl 1):S55-S58

9. Jennett B, Snoek J, Bond MR, et al: Disability after severe head injury: Observations on the use of the Glasgow Outcome Scale. J Neurol Neurosurg Psychiatry 1981; 44:285-293
10. Steyerberg EW, Mushkudiani N, Perel P, et al: Predicting outcome after traumatic brain injury: Development and international validation of prognostic scores based on admission characteristics. PLoS Med 2008; 5:e165

11. Andrews PJ, Sinclair LH, Harris B, et al; Eurotherm3235Trial collaborators: Study of therapeutic hypothermia $\left(32\right.$ to $\left.35^{\circ} \mathrm{C}\right)$ for intracranial pressure reduction after traumatic brain injury (the Eurotherm3235Trial): Outcome of the pilot phase of the trial. Trials 2013; 14:277

12. Perel $\mathrm{P}$, Arango $\mathrm{M}$, Clayton $\mathrm{T}$, et al; MRC CRASH Trial Collaborators: Predicting outcome after traumatic brain injury: Practical prognostic models based on large cohort of international patients. BMJ 2008; 336:425-429

13. Roozenbeek B, Lingsma HF, Lecky FE, et al; International Mission on Prognosis Analysis of Clinical Trials in Traumatic Brain Injury (IMPACT) Study Group; Corticosteroid Randomisation After Significant Head Injury (CRASH) Trial Collaborators; Trauma Audit and Research Network (TARN): Prediction of outcome after moderate and severe traumatic brain injury: External validation of the International Mission on Prognosis and Analysis of Clinical Trials (IMPACT) and Corticoid Randomisation After Significant Head injury (CRASH) prognostic models. Crit Care Med 2012; 40:1609-1617

14. Panczykowski DM, Puccio AM, Scruggs BJ, et al: Prospective independent validation of IMPACT modeling as a prognostic tool in severe traumatic brain injury. J Neurotrauma 2012; 29:47-52

15. Carney N, Totten AM, O'Reilly C, et al: Guidelines for the Management of Severe Traumatic Brain Injury, Fourth Edition. Neurosurgery 2017; 80:6-15

16. Chesnut RM, Temkin N, Carney N, et al; Global Neurotrauma Research Group: A trial of intracranial-pressure monitoring in traumatic brain injury. N Engl J Med 2012; 367:2471-2481

17. Cremer OL, van Dijk GW, van Wensen E, et al: Effect of intracranial pressure monitoring and targeted intensive care on functional outcome after severe head injury. Crit Care Med 2005; 33:2207-2213

18. Cooper DJ, Rosenfeld JV, Murray L, et al; DECRA Trial Investigators; Australian and New Zealand Intensive Care Society Clinical Trials Group: Decompressive craniectomy in diffuse traumatic brain injury. N Engl J Med 2011; 364:1493-1502

19. Roberts I, Yates D, Sandercock P, et al; CRASH trial collaborators: Effect of intravenous corticosteroids on death within 14 days in 10008 adults with clinically significant head injury (MRC CRASH trial): Randomised placebo-controlled trial. Lancet 2004; 364: 1321-1328

20. Clifton GL, Valadka A, Zygun D, et al: Very early hypothermia induction in patients with severe brain injury (the National Acute Brain Injury Study: Hypothermia II): A randomised trial. Lancet Neurol 2011; 10:131-139

21. Hutchison JS, Ward RE, Lacroix J, et al; Hypothermia Pediatric Head Injury Trial Investigators and the Canadian Critical Care Trials Group: Hypothermia therapy after traumatic brain injury in children. $N$ Engl J Med 2008; 358:2447-2456

22. Hutchinson PJ, Kolias AG, Timofeev IS, et al; RESCUEicp Trial Collaborators: Trial of decompressive craniectomy for traumatic intracranial hypertension. N Engl J Med 2016; 375:1119-1130

23. Clifton GL, Miller ER, Choi SC, et al: Lack of effect of induction of hypothermia after acute brain injury. N Engl J Med 2001; 344: 556-563

24. Maekawa T, Yamashita S, Nagao S, et al; Brain-Hypothermia Study Group: Prolonged mild therapeutic hypothermia versus fever control with tight hemodynamic monitoring and slow rewarming in patients with severe traumatic brain injury: A randomized controlled trial. J Neurotrauma 2015; 32:422-429 